\title{
Test Administration Methods and Cognitive Test Scores in Older Adults with Hearing Loss
}

\author{
Jing Shen ${ }^{\mathrm{a}}$ Melissa Sherman ${ }^{\mathrm{b}}$ Pamela E. Souza $^{\mathrm{c}}$ \\ aDepartment of Speech, Language and Hearing Sciences, Western Michigan University, Kalamazoo, MI, \\ USA; ${ }^{b}$ Compass Health Center, Chicago, IL, USA; ${ }^{C}$ Department of Communication Sciences and Disorders, \\ Northwestern University, Evanston, IL, USA
}

\section{Keywords}

Aging $\cdot$ Hearing loss $\cdot$ Cognitive impairment $\cdot$ Cognitive screening

\begin{abstract}
In light of the high prevalence of hearing loss and cognitive impairment in the aging population, it is important to know how cognitive tests should be administered for older adults with hearing loss. The purpose of the present study is to examine this question with a cognitive screening test and a working memory test. Specifically, we asked the following questions in 2 experiments. First, does a controlled amplification method affect cognitive test scores? Second, does test modality (visual vs. auditory) impact cognitive test scores? Three test administration conditions were created for both Montreal Cognitive Assessment (MoCA) and working memory test (a word recognition and recall test): auditory amplified, auditory unamplified, and visual. The auditory administration was implemented through a computer program to control for presentation level while the visual condition was implemented through timed computer slides. Data were collected from older individuals with mild-tomoderate sensorineural hearing loss. We did not find any effect of amplification or test modality on the total score of the cognitive screening test (i.e., MoCA). Amplification improved
\end{abstract}

working memory performance as measured by word recall performance, but test modality (auditory vs. visual) did not. These results are consistent with literature in demonstrating a downstream effect of audibility on working memory performance. From a clinical perspective, these findings are informative for developing clinical administration protocols of these tests for older individuals with hearing loss.

(c) 2019 S. Karger AG, Basel

\section{Introduction}

Age-related hearing loss is highly prevalent in the aging cohort $[1,2]$. According to national survey data, the prevalence of hearing loss is $49 \%$ in the age group of $60-69$ years old [1] and 63\% in those who are 70 years and older [3]. While the hearing aid adoption rate varies from 3.4 to $40 \%$ depending on hearing loss severity [3], the majority of these hearing loss cases remain untreated. Another health condition that also has a strong impact on the aging population is mild cognitive impairment (MCI). MCI is a clinical label used for describing decline in one or more cognitive abilities that is severe enough to raise clinical concern without impacting functional activities. The prevalence of MCI in most world regions is approximately $20-40 \%$ in older adults above 65 years [ 4 ] and $25 \%$

\section{KARGER}

(c) 2019 S. Karger AG, Basel

E-Mail karger@karger.com

www.karger.com/ger
Jing Shen

Department of Speech, Language and Hearing Sciences

Western Michigan University, 1903 W. Michigan Ave.

Kalamazoo, MI 49008 (USA)

E-Mail jing.shen@wmich.edu 
for community dwelling older adults in the United States [5-7]. While not all cases of MCI will progress to dementia, early detection and diagnosis have been shown to be critical in successful intervention [8]. Due to the high prevalence of hearing loss and cognitive impairment, many older individuals may cope with hearing loss and cognitive difficulties at the same time. There is evidence from both epidemiological and laboratory research that hearing loss is associated with cognitive difficulty and impairment $[9,10]$. Clinically, many older individuals who seek health care may have both untreated hearing loss and undiagnosed cognitive difficulties.

\section{Amplified Test Administration and Cognitive \\ Screening Results}

As there are various benefits to supporting a more regular administration of cognitive screening by first-line health-care providers such as audiologists [11], it is important to first ensure that the screening result is not compromised by reduced audibility or signal degradation due to hearing loss. Therefore, one crucial question that should be answered is whether older individuals who have hearing loss may be more likely to fail cognitive screening tests because of their hearing difficulty. In other words, they either cannot hear the test instruction and material well or have to expend effort/cognitive resources to the instructions and spoken items that may reduce recall or processing performance on the test items.

Researchers have examined this question with a variety of approaches. One study that was done on older individuals who have hearing and vision impairment tried removing those test items that heavily rely on auditory processing and found this modification had a significant effect on screening scores [12]. Specifically, more individuals with hearing loss passed the screening test (i.e., Montreal Cognitive Assessment [MoCA] [13]) when those items were removed, as compared to in the original version. Using the Mini-Mental State Examination [14], another study investigated the impact of simulated hearing loss on cognitive screening results [15]. They found that reduced audibility significantly affected test performance in a group of young adults who had normal hearing and were free of cognitive concerns. Further evidence came from a recent study that tested older listeners with either mild-to-moderate hearing loss or normal hearing [16]. All participants were tested twice using different versions of MoCA. Those with normal hearing were tested unaided twice, and those with hearing loss were tested once aided (with hearing aids or personal amplification) and once unaided. Consistent with previous findings [12,

Cognitive Test Methods in Older Adults with Hearing Loss
17], older individuals with hearing loss had lower cognitive test scores compared to their peers with normal hearing. While the amplification improved speech recognition performance, its effect on MoCA performance was not significant.

The evidence so far has been in favor of the hypothesis that individuals with hearing loss, as a group, have a "genuinely" lower cognitive performance than their peers with good hearing $[9,10,16]$. In other words, their lower scores of cognition tests cannot be attributed to any difficulty/effort in auditory processing during test administration. Nevertheless, it is worth a thorough probe to make sure the variability from the amplification was properly controlled in previous work. We know most listeners in the previous study [18] used their own hearing aids and the test was administered via live voice. As hearing aid processing can distort sound signal and negatively affect speech intelligibility, the method could introduce variability in sound level and quality $[19,20]$. Therefore, potential adverse signal distortion may offset the benefit from improved audibility, with the consequence of worse performance on a cognitive measure. To test this possibility, a more controlled amplification method or a visual administration that does not rely on auditory processing should be implemented for examining the potential impact of audibility on test scores. The first objective of Experiment 1 is to determine whether a more controlled amplification method has an effect on cognitive screening scores in older individuals with hearing loss.

\section{Amplified Test Administration and Working Memory Performance}

While a previous study showed a null effect of amplification on cognitive screening test results [16], one possibility is that MoCA, similar to other screening tests, is so brief in each subdomain of cognitive functions (with only 1 or 2 test items per subdomain) that it reduces the power to show any effect of auditory processing difficulty. As this brevity is certainly necessary for cognitive screening tests, it may also lead to reduced variability on each measure of cognitive functions, particularly when the perceptual effect of hearing loss is of interest. Therefore, a cognitive test that measures cognitive ability on a specific subdomain and is based on a greater number of test items can be used to examine a potential impact of amplification and test modality on performance. For example, a recent study examined performance on a verbal learning test (with words as test material) across 2 age-matched groups of older listeners. One group had hearing loss and the other group had normal hearing [21]. The data 
demonstrated a significant impact of audibility on test performance in an auditory version. Older individuals with hearing loss performed worse than those with normal hearing when test material was presented using a conversational level ( $60 \mathrm{~dB}$ SPL), while the reversed pattern was observed when the normal hearing group was tested with simulated hearing loss and the hearing loss group was tested with amplification. This finding suggests, when cognitive testing involves substantial auditory processing, the extra effort due to compromised audibility can influence the cognitive performance measured by the test.

Working memory capacity, defined as the ability to process and store information simultaneously [22], is one of the cognitive functions that are critical for daily functioning of older adults. Recent research has suggested a connection between working memory capacity and older listeners' speech perception under adverse conditions [23] and individual benefit from speech enhancement strategies [24]. Clinically, it has been argued that measuring working memory capacity in audiology service can provide important information for customizing and evaluating rehabilitative interventions [25]. An example of auditory working memory test is the recently developed Word Auditory Recognition and Recall Measure [26]. In the original study, a raised presentation level of 70-80 dB SPL has ensured a good recognition with average accuracy $80 \%$ for older listeners with hearing loss [26]. However, it remains a question what would be the consequence if the test is not administered with a properly adjusted presentation level and the audibility of stimuli is compromised. We know that compromised audibility has an impact on recall of speech material as a downstream effect of increased effort in perceiving speech [27, 28]. Specifically related to the speech recognition and recall test that is intended to be used in clinical settings, it is critical to show the importance of audibility in test administration. To serve this purpose, Experiment 1 included a word recognition and recall test administered with 2 auditory test conditions: one with a presentation level of $65 \mathrm{~dB}$ (which simulates a normal conversation level); and one with individually amplified presentation levels for older individuals with hearing loss (using NAL frequency shaping). The difference in performance between the 2 conditions can provide information regarding how this type of test should be administered in clinical setting.

To summarize, Experiment 1 had 2 objectives:

1 To determine whether a controlled amplification method has an effect on scores of a cognitive screening test (MoCA) in older individuals with hearing loss.
2 To determine whether a controlled amplification method has an effect on scores of an auditory working memory test in older individuals with hearing loss.

\section{Test Modality and Cognitive Screening Results}

In the search for an administration method that can measure the "true" cognitive status in individuals with hearing loss, a solution that has been considered is visual administration of cognitive screening tests. Recent development of a visually administered version of MoCA aimed to use it for individuals with severe hearing loss and candidates for cochlear implants [29]. In that study, MoCA was converted to a timed PowerPoint presentation and given to 2 groups of older adults (one with normal hearing, one with severe hearing loss). Overall, the effect was likely not strong enough to impact the screening results as the difference in MoCA total score was $<1$ point between the 2 versions with different modalities. Therefore, for those patients with severe hearing loss, a visually administered cognitive screening test provides a viable clinical option. However, with those older individuals with mild-to-moderate hearing loss, would visual administration be a better choice as compared to an amplified auditory administration?

Experiment 2 of the present study aimed to examine this question by comparing MoCA scores with visual and auditory administrations in older adults with hearing loss. Specifically, the questions we asked were (1) for older listeners with mild-to-moderate hearing loss, could difficulty in auditory perception lead to any difference between visual and unamplified auditory administrations? (2) does amplification in an auditory administration significantly improve performance as compared to visual administration?

\section{Test Modality and Working Memory Performance}

As discussed earlier, it is worth noting that a cognitive screening test such as MoCA may be too brief with a mix of test items that taxes both visual/auditory modalities, and therefore the comparison between different test modalities may only be partially affected by the effort in auditory processing experienced by listeners with hearing loss. Following this rationale, we were interested in the modality effect (i.e., auditory versus visual administration) on an auditory working memory test, which should rely more heavily on auditory processing as compared to a cognitive screening test. While previous data have demonstrated that older individuals with hearing loss underperform their peers with good hearing in cognitive tests that were given in the auditory modality, the findings were largely reported as comparison between groups. For example, a previous study has examined the recall performance of in- 
dividuals with mild hearing loss using word lists presented through visual versus auditory modality [30]. The results showed that, in auditory administration, individuals with even mild hearing loss did worse with the recall task than the age-matched controls with good hearing, while the performance of 2 groups was comparable in visual administration. Similar results were shown in a recent study that used a clinical test of verbal learning [20]. Wong et al. [20] showed that while older individuals with hearing loss performed equally well compared to their normal hearing peers on a visual administration of the test, performance in auditory administration was strongly affected by audibility. In a study that used a n-back digit recall test, Guerreiro et al. [17] found an inferior performance that was independent of test modality in older individuals with hearing loss, as compared to their peers with good hearing. This finding is different from other studies [20,30] and is likely due to the difference in the tasks employed. Recognition of digits does not rely on auditory perception as heavily as word recognition does. Therefore, the effect of test modality is less prominent in the n-back digit recall task, as compared to the word recall tasks.

While the previous data provide evidence for the effect of test modality on performance in a working memory test, the findings were largely based on comparisons across different age-matched groups. From a clinical perspective, if the goal is to determine how to measure an individual's working memory using a word recall test, it is more informative to have data by comparing the performance across different conditions within the same group of participants, to control for individual variability in working memory. Therefore, one of the aims of Experiment 2 was to compare word recall performance across visual and auditory administrations in a group of older listeners with mild-to-moderate hearing loss. Importantly, the stimuli were word lists that are frequently used in audiology clinical settings to measure speech perception.

To summarize, Experiment 2 had 4 objectives:

1 To determine whether there is any difference between visual and unamplified auditory administrations of a cognitive screening test (MoCA) in a group of older individuals with mild-to-moderate hearing loss.

2 To determine whether there is any difference between visual and amplified auditory administrations of a cognitive screening test (MoCA) in a group of older individuals with mild-to-moderate hearing loss.

3 To determine whether there is any difference between visual and unamplified auditory administrations of an auditory working memory test in a group of older individuals with mild-to-moderate hearing loss.
Table 1. Pure tone thresholds (average of $0.5,1$, and $2 \mathrm{kHz}$, in $\mathrm{dB}$ $\mathrm{HL}$ ) of the 3 groups of older participants with hearing loss

\begin{tabular}{|c|c|c|}
\hline $\begin{array}{l}\text { Group } 1 \\
\text { (Experiment 1): } \\
\text { auditory amplified } \\
\text { vs. unamplified }\end{array}$ & $\begin{array}{l}\text { Group } 2 \\
\text { (Experiment 2): } \\
\text { visual vs. auditory } \\
\text { amplified }\end{array}$ & $\begin{array}{l}\text { Group } 3 \\
\text { (Experiment 2): } \\
\text { visual vs. auditory } \\
\text { unamplified }\end{array}$ \\
\hline 36.67 & 43.33 & 43.33 \\
\hline 56.67 & 55 & 43.33 \\
\hline 33.33 & 36.67 & 43.33 \\
\hline 41.67 & 51.67 & 36.67 \\
\hline 48.33 & 38.33 & 48.33 \\
\hline 36.67 & 41.67 & 48.33 \\
\hline 35 & 41.67 & 30 \\
\hline 36.67 & 40 & 38.33 \\
\hline 40.62 & 43.54 & 41.45 \\
\hline
\end{tabular}

The last row is the average of the participants in each group.

4 To determine whether there is any difference between visual and amplified auditory administrations of an auditory working memory test in a group of older individuals with mild-to-moderate hearing loss.

\section{Experiment 1}

\section{Methods}

Participants

Eight older adults (4 female and 4 male) aged 70-84 years (mean age 77 years) participated in this experiment. Using the PowerSim function in R [31], a power level of above $80 \%$ was confirmed for a small effect size $(b=0.1)$ in a linear mixed effect model with 8 participants, 2 test conditions and 10 test items (for both $\mathrm{MoCA}$ and the working memory test). Inclusion criteria were symmetrical mild-to-moderate sensorineural hearing loss; a difference in pure-tone average $(0.5,1,2 \mathrm{kHz}) \leq 10 \mathrm{~dB}$ across ears; and airbone gaps $\leq 10 \mathrm{~dB}$. The participant's audiometric thresholds were reported in Table 1 . The participants were recruited via newspaper advertisements and flyers in the Greater Chicago area. All participants were native English speakers and reported good general health. They had either normal or corrected-to-normal vision, screened using a Snellen Chart. Dementia Rating Scale II [32] was used to measure general cognitive functioning and screen for dementia. None of the participants scored below 132, which corresponds to the 30th percentile in the age-matched norm (Lucas et al. [33], 1998) and a cutoff score that has high accuracy for screening MCI [34].

\section{Stimuli and Procedure}

A verbal administration of the MoCA [13] was recorded by one of the authors (M.S.), who has a local accent that is familiar to Chicago residents. The recording was segmented into individual items/questions and normalized for presentation level. 
For the word recognition and recall test, 2 NU-6 wordlists [35] (list $2 \mathrm{~A}$ and $3 \mathrm{~A}$ ) were used with 42 words in each list. The 2 lists were matched for lexical frequency and neighborhood density [36]. The test was designed using the same structure as Word Auditory Recognition and Recall Measure [26] with set size from 2 to 6 . The words were carefully arranged into sets so there was no explicit semantic/phonological relationship between any words in each set. There were 2 runs per set size. A 2-word set was used as practice run prior to the first test set.

The tests were all administered using customized MATLAB programs (Natick, MA, USA). All the recorded material was calibrated to have averaged output level of $65 \mathrm{~dB}$ SPL for the unamplified condition. In the amplified condition, the speech stimuli were amplified using the National Acoustics Laboratories-Revised linear prescriptive formula based on individual thresholds averaged across 2 ears [37]. Stimuli were presented binaurally using an M-Audio FastTrackPro external soundcard (M-Audio) and a Sennheiser HD-25 II headphone (Wedemark, Germany). Listeners were seated in a double-wall sound proofed booth with a tester who controlled the pace of the administration. All the instruction and stimuli was presented over earphones and the tester did not have any verbal interaction with the participant.

For MoCA, participants were instructed to write down the answer on a response sheet for the visuospatial items and respond verbally for the rest of the test. The tester recorded the answers and scored afterwards. For the word recognition and recall test, each participant was tested with one list per condition, with list order and condition order counterbalanced. The participants were instructed to complete a few tasks in this test. First, they were asked to repeat back each word immediately after the word is played, followed by indicating verbally which half of the alphabet the first letter of the word is from. After all the words in each set are played, they were asked to recall as many words as possible from the set. The recall performance was scored independent of the recognition performance. In other words, if the participant repeated an incorrect word but still recalled the same word later, it was counted as correct.

To verify the effect of amplification on speech perception, 2 speech recognition tests were included with recorded stimuli from the same talker, a word recognition test using 2 lists of W22 [38] (50 words per list), and a sentence recognition test using 2 lists of Perceptually Robust English Sentence Test Open-set (PRESTO) sentences [39] (with 18 sentences per list). All speech stimuli were presented with the same protocols as the MoCA stimuli. These 2 tests were administered to all the older participants in Experiments 1 and 2 , and data were collapsed before analysis.

\section{Results}

MoCA

Data analysis was conducted using mixed-effects linear regression using R (R Core Team-Version 3.2.1). Mixed effects linear regressions were conducted using R's lme4 [40] and lmerTest libraries [41]. The models included fixed effects of amplification conditions (amplified vs. unamplified), test version/list \# (version A vs. B for MoCA), and test order (1st vs. 2nd session), as well as random by-participant and by-item intercepts. While the variable of interest was test condition, test version and order were both included to control for any version/order effect. All the categorical variables were contrast coded using simple coding strategy.
The results showed that there was no effect of amplification as the average total score stayed the same across the 2 conditions (24.125 points out of 30 points total, SD: amplified 1.69 points, unamplified 1.26 points). Neither version nor order had a significant impact on test score.

\section{Word Recall Performance}

For word recognition, the models included fixed effects of amplification conditions (amplified vs. unamplified), test version/list \# (list 2 vs. 3 for word recall test), test order (1st vs. 2nd session), item set size (2-6), as well as random by-participant intercepts. There was a significant effect of amplification on recognition performance, with an improvement from 73 to $93 \%$ in the amplified condition as compared to the unamplified condition $(b=0.2, t$ $(145)=6.13, p<0.001)$.

For word recall, the models included fixed effects of amplification conditions (amplified vs. unamplified), test version/list \# (List 2 vs. 3 for word recall test), test order (1st vs. 2 nd session), item set size (2-6), recognition performance, as well as random by-participant intercepts. Recall performance was significantly better with amplification (mean accuracy of $69.06 \%$ ) than without it (mean accuracy of $65.93 \%, b=0.07, t(148)=2.33, p<0.05)$ when the recognition was controlled for.

\section{Amplification Effect on Speech Recognition}

The same model structure was used for verifying amplification effect on speech recognition performance, with fixed factors being test condition (amplified vs. unamplified), test list, and order. Both datasets, the word recognition test (W22) and sentence recognition test (PRESTO), showed a strong effect of amplification on speech recognition. Test condition (amplified vs. unamplified) was a significant factor that impacted on model fit, while order and test list were not. Amplification significantly improved speech recognition with W22 $(\mathrm{b}=0.24, t(23)=6.74, p<0.001)$ and PRESTO sentences $(b=0.69, t(22)=2.83, p<0.01)$.

\section{Experiment 2}

\section{Methods}

Participants

Sixteen older adults with hearing loss (6 female and 10 male, age range $58-80$ years, mean age 73.5 years) participated in this experiment. None of them participated in the Experiment 1. The older group had the same inclusion criteria as in Experiment 1. Their audiometric thresholds are presented in Table 1.

Stimuli and Procedure

A visual administration of MoCA was created as timed slides using Microsoft PowerPoint (Redmond, WA, USA). All the instructions were visually presented on the slides using 32-pt Calibri font. The instruction slides were self-paced with a time limit depending on the length of the text. The presentation of the stimuli (e.g., words, numbers, and letters) was timed to closely match the auditory presentation ( $2 \mathrm{~s}$ per word/number/letter). The timing was first pilot tested to make sure the visual presentation of stimuli was timed appropriately.

A visual version of the word recognition and recall test was also included using PowerPoint slides. The instructions were presented 


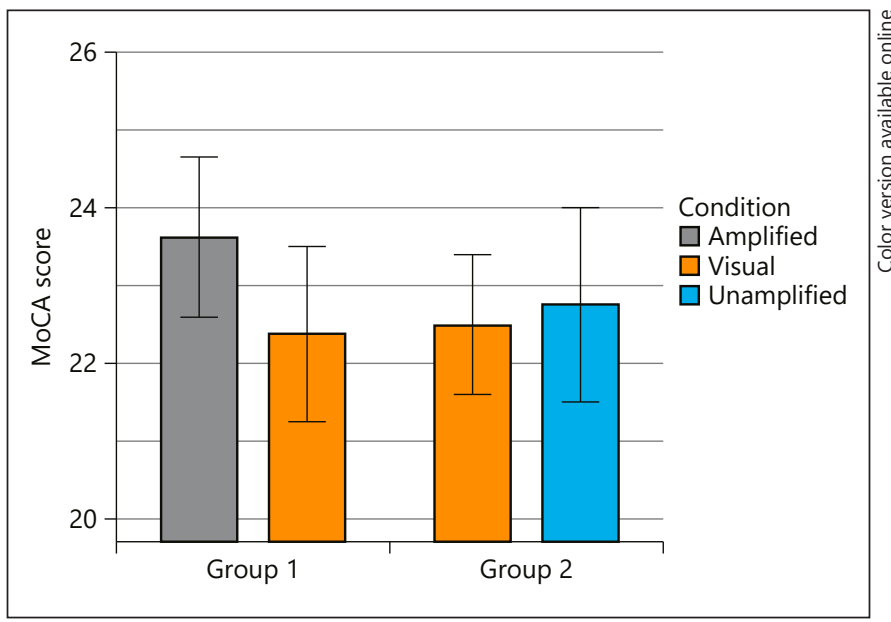

Fig. 1. MoCA total scores of the 2 groups (Group 1: amplified vs. visual, Group 2: unamplified vs. visual. Error bars present $\pm 1 \mathrm{SE}$ ). MoCA, Montreal Cognitive Assessment.

on the first 2 slides for the participants to read before any clarification questions were answered. A 2-word practice run was completed prior to the formal test. The test used exactly the same protocol as the auditory administration, with set size increasing from 2 to 6 . Each word was presented on a PowerPoint slide with 60-pt Calibri font. The participants were instructed to repeat back the word on the slide verbally and also indicate which half of the alphabet the first letter of the word was from. After each set, an instruction was presented on the slide to prompt recall. The presentation of the slides was controlled by the tester. The word recall performance was scored independent of the recognition performance to minimize the impact of the inherently different recognition accuracy across modality.

The 16 older individuals with hearing loss were separated into 2 groups that were matched in gender and age (both have 5 males and 3 females, mean age 73.4 and 73.8 years, respectively). For Group 1, visual and amplified auditory conditions were given. For Group 2, visual and unamplified auditory conditions were given. Both older groups had auditory and visual conditions separated in 2 testing sessions. The order of condition and version was fully counterbalanced. The auditory administration followed the same protocol as in Experiment 1.

\section{Results}

\section{MoCA}

Linear mixed effect models were built on this dataset using the same structure as in Experiment 1. For both older groups, test condition did not significantly improve model fit (amplified vs. visual: $\mathrm{b}=0.1, t(140)=1.04, p>$ 0.1 ; unamplified vs. visual: $\mathrm{b}=0.02, t(140)=0.19, p>0.1)$. This means total test scores were not significantly different across visual and auditory conditions. Performance scores are plotted in Figure 1.

Cognitive Test Methods in Older Adults with Hearing Loss

\section{Word Recognition and Recall Performance}

Data from the 2 older groups showed that recognition performance in the visual administration was at perfect levels for all individuals and was also relatively high in the amplified auditory condition (with above 90\% group mean of correct recognition) and the unamplified auditory condition (with 70-80\% group mean of correct recognition).

As to recall performance, no significant difference was observed across modality conditions (visual vs. amplified: $b=0.02, t(144)=0.86, p>0.1$; visual vs. unamplified: $b=-0.01, t(144)=-0.28, p>0.1)$, even with recognition performance controlled for.

\section{General Discussion}

\section{Amplified versus Unamplified Administration of Cognitive Screening Tests}

The present study first examined the question whether a controlled amplification protocol has an effect on scores of cognitive tests, including a cognitive screening test (MoCA) and a working memory (word recognition and recall) test. Consistent with the previous finding that amplification provided by hearing aids did not significantly affect cognitive screening test scores [16], results from the present study showed no effect of amplification (which, for consistency and control, was implemented using computer simulation of NAL frequency-gain instead of personal hearing aids) on the total scores of MoCA. In fact, the MoCA mean score remained the same across the amplified and unamplified conditions in this dataset. While amplification significantly improved speech recognition performance with speech material including words and sentences, the null effect of amplification on cognitive screening score indicates any speech recognition difficulty in the unamplified condition may not be substantial enough to influence cognitive performance that is captured by a brief screening test.

From a clinical perspective, this result suggests test administration without amplification does not significantly affect the test score for MoCA, and perhaps other brief cognitive screening tools. Taken together with the previous results that an unfamiliar amplification device (such as a clinic-provided pocket talker) actually worsened the performance [16], we think cognitive screening tests should be administered to older individuals with hearing loss using the condition that the client is most comfortable with. In other words, if an older individual with mild-to-moderate hearing loss 
does not use hearing aids, then it is a viable solution to administer the cognitive screening test without amplification, as long as no substantial difficulty was observed during conversation. This conclusion, however, does not mean that no caution should be taken in choosing a suitable test environment for giving a cognitive test. In fact, in the present study, both administrations of MoCA (amplified and unamplified) were done in a soundproofed booth with supra-aural headphones. This test setting ensured the absence of background noise that could interfere with speech perception and cognitive performance, and as a consequence lower the test score [42]. Every effort should be taken to have the test administered in a quiet and well-lit space to optimize auditory and visual perception.

It is worth noting, however, that the participants in the present study were recruited through voluntary response to study advertisement. This may lead to potential self-selection in hearing abilities. For example, we know this is a well-educated sample with the mean education of 16.75 years (with SD of 2.7 years). In other words, it is likely that these older individuals may have better speech perception and cognitive abilities (due to factors other than hearing) than those who did not choose to respond. Therefore, the null effect of amplification in this sample of older individuals may not generalize to an aging population that has more diversity. We are currently working on examining this possibility with the comparison between different racial/ethnic groups.

Further, it is important to note that the brevity of a cognitive screening test such as MoCA is likely to be a driving factor behind the null effect of amplification. This result may not generalize to other cognitive tests that have more test items and be more comprehensive (e.g., a neuropsychology test battery). In fact, more recent evidence has demonstrated the amplification effect in a verbal learning test that is frequently used in clinical neuropsychology [20]. Taken together with our finding of a significant effect of amplification on the word recall performance, this evidence highlights the importance for future work to examine the potential impact of amplification on cognitive test results using other tests that are longer and more comprehensive.

\section{Effect of Amplification on Word Recognition and \\ Recall Performance}

On the other hand, our investigation of the amplification effect on word recall has demonstrated a significant amplification effect on word recall performance. This ef- fect persisted when recognition performance was under control in the analyses. This finding has clinical implication by providing the information about how this type of working memory test should be administered to individuals with hearing loss. Specifically, the test material should be presented with an audible presentation level to every individual (with customized presentation level being ideal). Using headphone/earphone presentation is likely to help control audibility and also to eliminate any variability in signal level/quality introduced by amplification device (e.g., hearing aids). It is worth noting that the recognition accuracy was above $93 \%$ in the amplified condition but only $73 \%$ in the unamplified condition. While 73\% would be a low but acceptable word recognition performance in many clinical scenarios, it is evident that the effort in the auditory processing can have a downstream effect and negatively impact memory encoding and storage, which has been consistently demonstrated by previous studies $[28,32]$.

While the difference in recall performance being statistically significant, the effect size was nevertheless small $(b=0.07)$ with recall performance only about $5 \%$ lower in the unamplified condition as compared to the amplified one. From a cognitive processing perspective, this suggests a limited influence from audibility on this type of cognitive task. It is worth noting that Wong et al. [20] found that audibility appeared to have a substantial impact on a verbal learning test (with a medium to large effect size). Compared to our word recognition and recall test, the test used in Wong's study requires more semantic processing and has a longer delay before the recall. The contrast between the findings from these 2 studies suggests the audibility effect on cognitive performance is task specific and the effect is likely to be stronger with cognitive tasks that involve heavier cognitive processing load. This possibility should be investigated in future research.

There was a positive order effect in word recognition and recall performance, suggesting a practice effect with the 2 administrations if both were given through auditory administration. As the test conditions were counterbalanced (i.e., half participants had amplified condition first and the other half had opposite order) and the test order is also included in the model as a control variable, this practice effect is not likely to change the interpretation of the findings. This result, however, does highlight how strong and fast the practice effect occurs (consider the 2 administrations were at least over a week apart), which is important for future work to consider in the study design.
Shen/Sherman/Souza 


\section{Test Modality and Cognitive Test Scores}

The second objective of the present study was to compare performance across visual and auditory administrations for a cognitive screening test (MoCA) and a working memory test. Our finding on MoCA is consistent with the previous study by Lin et al. [29] in demonstrating a subtle difference ( $<1$ point) in total MoCA score between unamplified auditory and visual administrations. This result aligns well with the previous finding from Lin's study and also extends to populations beyond older group with good hearing.

It is worth noting that the group mean (for older hearing loss group) of the MoCA total score in the amplified condition was $>1$ point higher than in the visual condition (23.6 vs. 22.3 ). While this difference is not substantial and does not indicate a reliable change in cognitive abilities [43], it is possible that this difference of 1 point can place that individual either above or below a passing score. This is not surprising as the participants in our study have mild-to-moderate hearing loss and is a group that typically benefit from amplification (while the comparison in Lin's work was based on data from older adults with good hearing). While this finding should be verified using a larger dataset in future work, our current data indicate an auditory administration is likely to be more suitable for these older individuals as compared to a visually administered cognitive screening test.

Our working memory (i.e., word recall) data did not show a visual superiority effect over auditory administrations in the older groups. While visual administration presents an appealing alternative for older individuals with hearing loss, it is important to consider the difference in the construct validity between visual and auditory administrations. According to Baddeley's working memory model [44], the phonological loop is a critical component in the memory encoding process for this type of stimuli. As this process heavily relies on the heard and spoken speech, seeing the words instead of hearing them has reduced one step in memory encoding as the words were not presented verbally. The words have to be encoded only based on speech production (i.e., reading aloud). As a consequence, visual administration of the test differs from the auditory administration in terms of the cognitive construct being measured, even when signal presentations via both sensory modalities were optimized. Further, if we are interested in how well older individuals retain information from everyday speech communication, working memory measured through a visual modality

Cognitive Test Methods in Older Adults

with Hearing Loss is likely not serving the purpose due to the lack of construct validity.

Taken together with the significant amplification effect in Experiment 1, our findings suggest a highly audible material presentation is the key to appropriate administration of auditory working memory test for older individuals with mild-to-moderate hearing loss. For those individuals whose hearing loss is too severe to benefit from amplification (e.g., cochlear implant candidates), the solution of a visually administered working memory test should be examined in future work.

\section{Acknowledgement}

The authors thank Amanda Mueller and Rebecca Welles for assistance with data collection.

\section{Statement of Ethics}

The study protocol was reviewed and approved by Institutional Review Board at Northwestern University. Participants in this study have given their written informed consent.

\section{Disclosure Statement}

The authors have no conflicts of interest to declare.

\section{Funding Sources}

This work is supported by grants from National Institutes of Health (NIH R01DC012289 and F32 DC014629).

\section{Author Contributions}

J.S., M.S., and P.E.S.: designed the study. J.S.: collected and analyzed data. J.S., M.S., and P.E.S.: wrote the manuscript.

$\begin{aligned} & \text { References } \quad \begin{array}{l}\text { Agrawal Y, Platz EA, Niparko JK. Prevalence } \\ \text { of hearing loss and differences by demo- } \\ \text { graphic characteristics among US adults: data }\end{array} \\ & \text { from the National Health and Nutrition Ex- } \\ & \text { amination Survey, 1999-2004. Arch Intern } \\ & \text { Med. 2008 Jul;168(14):1522-30. } \\ & 2 \text { Cruickshanks KJ, Wiley TL, Tweed TS, Klein } \\ & \text { BE, Klein R, Mares-Perlman JA, et al.; The } \\ & \text { Epidemiology of Hearing Loss Study. Preva- } \\ & \text { lence of hearing loss in older adults in Beaver } \\ & \text { Dam, Wisconsin. Am J Epidemiol. 1998 Nov; } \\ & 148(9): 879-86 .\end{aligned}$


3 Lin FR, Thorpe R, Gordon-Salant S, Ferrucci L. Hearing loss prevalence and risk factors among older adults in the United States. J Gerontol A Biol Sci Med Sci. 2011 May;66(5): 582-90.

4 Ward A, Arrighi HM, Michels S, Cedarbaum JM. Mild cognitive impairment: disparity of incidence and prevalence estimates. Alzheimers Dement. 2012 Jan;8(1):14-21.

5 Petersen RC, Roberts RO, Knopman DS, Geda YE, Cha RH, Pankratz VS, et al.; The Mayo Clinic Study of Aging. Prevalence of mild cognitive impairment is higher in men. Neurology. 2010 Sep;75(10):889-97.

6 Katz MJ, Lipton RB, Hall CB, Zimmerman ME, Sanders AE, Verghese J, et al. Age-specific and sex-specific prevalence and incidence of mild cognitive impairment, dementia, and Alzheimer dementia in blacks and whites: a report from the Einstein Aging Study. Alzheimer Dis Assoc Disord. 2012 Oct-Dec; 26(4):335-43.

7 Manly JJ, Tang MX, Schupf N, Stern Y, Vonsattel JP, Mayeux R. Frequency and course of mild cognitive impairment in a multiethnic community. Ann Neurol. 2008 Apr;63(4): 494-506.

8 Prince M, Bryce R, Ferri C. World Alzheimer Report 2011: The benefits of early diagnosis and intervention [Internet]. 2011. Available from: https://www.alz.co.uk/research/worldreport-2011.

9 Lin FR, Yaffe K, Xia J, Xue QL, Harris TB, Purchase-Helzner E, et al.; Health ABC Study Group. Hearing loss and cognitive decline in older adults. JAMA Intern Med. 2013 Feb; 173(4):293-9.

10 Dawes P, Emsley R, Cruickshanks KJ, Moore DR, Fortnum H, Edmondson-Jones $\mathrm{M}$, et al. Hearing loss and cognition: the role of hearing AIDS, social isolation and depression. PLoS One. 2015 Mar; 10(3):e0119616.

11 Shen J, Anderson MC, Arehart KH, Souza PE. Using cognitive screening tests in audiology. Am J Audiol. 2016 Dec;25(4):319-31.

12 Dupuis K, Pichora-Fuller MK, Chasteen AL, Marchuk V, Singh G, Smith SL. Effects of hearing and vision impairments on the Montreal Cognitive Assessment. Neuropsychol Dev Cogn B Aging Neuropsychol Cogn. 2015; 22(4):413-37.

13 Nasreddine ZS, Phillips NA, Bédirian V, Charbonneau S, Whitehead V, Collin I, et al. The Montreal Cognitive Assessment, MoCA: a brief screening tool for mild cognitive impairment. J Am Geriatr Soc. 2005 Apr;53(4): 695-9.

14 Folstein MF, Folstein SE, McHugh PR. "Minimental state". A practical method for grading the cognitive state of patients for the clinician. J Psychiatr Res. 1975 Nov;12(3):189-98.
15 Jorgensen LE, Palmer CV, Pratt S, Erickson KI, Moncrieff D. The effect of decreased audibility on MMSE performance: A measure commonly used for diagnosing dementia. J Am Acad Audiol. 2016 Apr;27(4):311-23.

16 Saunders GH, Odgear I, Cosgrove A, Frederick MT. Impact of Hearing Loss and Amplification on Performance on a Cognitive Screening Test. J Am Acad Audiol. 2018 Jul/Aug; 29(7):648-55

17 Guerreiro MJ, Van Gerven PW. Disregarding hearing loss leads to overestimation of agerelated cognitive decline. Neurobiol Aging. 2017 Aug;56:180-9.

18 Besser J, Koelewijn T, Zekveld AA, Kramer SE, Festen JM. How linguistic closure and verbal working memory relate to speech recognition in noise-a review. Trends Amplif. 2013 Jun;17(2):75-93.

19 Keidser G, Brew C, Peck A. Proprietary fitting algorithms compared with one another and with generic formulas. Hear J. 2003;56(3):2838.

20 Killion M. Myths about hearing aid benefit and satisfaction. Hearing Review. 2004;11(9): $12-7$

21 Wong CG, Rapport LJ, Billings BA, Ramachandran V, Stach BA. Hearing loss and verbal memory assessment among older adults. Neuropsychology. 2019 Jan;33(1):47-59.

22 Baddeley A. Working memory. Science. 1992 Jan;255(5044):556-9.

23 Akeroyd MA. Are individual differences in speech reception related to individual differences in cognitive ability? A survey of twenty experimental studies with normal and hearing-impaired adults. Int J Audiol. 2008 Nov; 47(2 Suppl 2):S53-71.

24 Shen J, Souza PE. On Dynamic Pitch Benefit for Speech Recognition in Speech Masker. Front Psychol. 2018 Oct 22;9:1967.

25 Pichora-Fuller MK. Cognitive aging and auditory information processing. Int $\mathrm{J}$ Audiol. 2003 Jul;42(Suppl 2):2S26-32.

26 Smith SL, Pichora-Fuller MK, Alexander G. Development of the word auditory recognition and recall measure: a working memory test for use in rehabilitative audiology. Ear Hear. 2016 Nov/Dec;37(6):e360-76.

27 Rabbitt PM. Channel-capacity, intelligibility and immediate memory. Q J Exp Psychol. 1968 Aug;20(3):241-8.

28 Wingfield A, Tun PA. Spoken Language Comprehension in Older Adults: Interactions between Sensory and Cognitive Change in Normal Aging. Semin Hear. 2001;22(03): 287-302.

29 Lin VY, Chung J, Callahan BL, Smith L, Gritters N, Chen JM, et al. Development of cognitive screening test for the severely hearing impaired: hearing-impaired MoCA. Laryngoscope. 2017 May;127(Suppl 1):S4-11.
30 Rabbitt P. Mild hearing loss can cause apparent memory failures which increase with age and reduce with IQ. Acta Otolaryngol Suppl. 1990;476:167-75; discussion 176.

31 Green P, MacLeod CJ. SIMR: an R package for power analysis of generalized linear mixed models by simulation. Methods Ecol Evol. 2016;7(4):493-8.

32 Jurica PJ, Leitten CL, Mattis S. DRS-2 dementia rating scale-2: professional manual. Psychological Assessment Resources; 1988.

33 Lucas JA, Ivnik RJ, Smith GE, Bohac DL, Tangalos EG, Kokmen E, et al. Normative data for the Mattis dementia rating scale. J Clin Exp Neuropsychol. 1998 Aug;20(4):536-47.

34 Green RC, Woodard JL, Green J. Validity of the Mattis Dementia Rating Scale for detection of cognitive impairment in the elderly. J Neuropsychiatry Clin Neurosci. 1995;7(3): 357-60.

35 Tillman TW, Carhart R. An expanded test for speech discrimination utilizing CNC monosyllabic words. Northwestern University Auditory Test No. 6. SAM-TR-66-55. Tech Rep SAM-TR. 1966 Jun:1-12.

36 Kučera H, Francis WN. Computational analysis of present-day American English. Dartmouth Publishing Group; 1967.

37 Byrne D, Dillon H, Ching T, Katsch R, Keidser G. NAL-NL1 procedure for fitting nonlinear hearing aids: characteristics and comparisons with other procedures. J Am Acad Audiol. 2001 Jan;12(1):37-51.

38 Hirsh IJ, Davis H, Silverman SR, Reynolds EG, Eldert E, Benson RW. Development of materials for speech audiometry. J Speech Hear Disord. 1952 Sep;17(3):321-37.

39 Gilbert JL, Tamati TN, Pisoni DB. Development, reliability, and validity of PRESTO: a new high-variability sentence recognition test. J Am Acad Audiol. 2013 Jan;24(1):26-36.

40 Bates D, Maechler M, Bolker B, Walker S. lme4: Linear mixed-effects models using Eigen and S4. R package version. 2014;1(7):123.

41 Kuznetsova A, Brockhoff PB, Christensen RHB. lmerTest: Tests for Random and Fixed Effects for Linear Mixed Effect Models (lmer Objects of lme4 Package). 2013.

42 Dupuis K, Marchuk V, Pichora-Fuller MK Noise affects performance on the Montreal Cognitive Assessment. Can J Aging. 2016 Sep; 35(3):298-307.

43 Kopecek M, Bezdicek O, Sulc Z, Lukavsky J, Stepankova H. Montreal Cognitive Assessment and Mini-Mental State Examination reliable change indices in healthy older adults. Int J Geriatr Psychiatry. 2017 Aug;32(8):86875.

44 Baddeley A. Working memory: theories, models, and controversies. Annu Rev Psychol. 2012;63(1):1-29. 